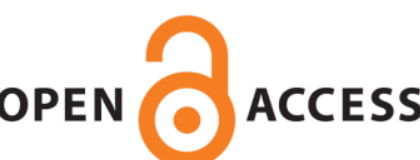

OPEN ACCESS

UWS Academic Portal

\title{
Analysis of foot kinematics wearing high heels using the Oxford Foot Model
}

Wang, Meizi ; Gu, Yaodong; Baker, Julien

Published in:

Technology and Health Care

DOI:

10.3233/THC-181264

Published: 07/05/2018

Document Version

Peer reviewed version

Link to publication on the UWS Academic Portal

Citation for published version (APA):

Wang, M., Gu, Y., \& Baker, J. (2018). Analysis of foot kinematics wearing high heels using the Oxford Foot Model. Technology and Health Care, 26(5), 815-823. https://doi.org/10.3233/THC-181264

\section{General rights}

Copyright and moral rights for the publications made accessible in the UWS Academic Portal are retained by the authors and/or other copyright owners and it is a condition of accessing publications that users recognise and abide by the legal requirements associated with these rights. 


\title{
Analysis of foot kinematics wearing high heels using the Oxford Foot Model
}

\author{
Wang, Meizi ; Gu, Yaodong; Baker, Julien S
}

\begin{abstract}
Wearing high heels is thought to lead to various foot disorders and injuries such as metatarsal pain, Achilles tendon tension, plantar fasciitis and haglund malformation. However, there is little available information explaining the specific mechanisms and reasons why wearing high heels causes foot deformity. Therefore, the purpose of this study was to investigate the foot kinematics of high heel wearers and compare any differences with barefoot individuals using the Oxford Foot Model (OFM). Fifteen healthy women aged 20-25 years were measured while walking barefoot and when wearing high heels. All subjects had little experience of wearing high heels when walking. The peak value of angular motion for the hallux with respect to the forefoot, the forefoot with respect to the hind foot, and the hind foot with respect to the tibia were all analyzed. Compared to the barefoot, participants wearing high heels demonstrated larger hallux dorsiflexion $\left(22.55^{\circ} \pm 1.62^{\circ}\right.$ VS $26.6^{\circ} \pm 2.33^{\circ}$ for the barefoot; $\mathrm{P}=0.001$ ), and less hallux plantarflexion during the initial stance phase $\left(-4.86^{\circ} \pm 2.32^{\circ} \mathrm{VS}-8.68^{\circ} \pm 1.13^{\circ} ; \mathrm{P}<0.001\right)$. There were also greater forefoot abduction $\left(16.15^{\circ} \pm 1.37^{\circ}\right.$ VS $\left.13.18^{\circ} \pm 0.79^{\circ} ; \mathrm{P}<0.001\right)$, but no significant differences were found in forefoot adduction between the two conditions. The hind foot demonstrated a larger dorsiflexion in the horizontal plane $\left(16.59^{\circ} \pm 1.69^{\circ} \mathrm{VS} 12.08^{\circ} \pm 0.9^{\circ} ; \mathrm{P}<0.001\right)$, greater internal rotation $\left(16.72^{\circ} \pm\right.$ $0.48^{\circ}$ VS $\left.7.97^{\circ} \pm 0.55^{\circ} ; \mathrm{P}<0.001\right)$, and decreased peak hind foot extension rotation $\left(-5.49^{\circ} \pm 0.69^{\circ}\right.$ VS $-10.73^{\circ} \pm 0.42^{\circ} ; \mathrm{P}=0.001$ ). This study is the first of its kind to investigate, in detail, foot motion kinematics when wearing high heels during walking. These findings complement existing kinematic evidence that wearing high heels can lead to foot deformities and injuries.
\end{abstract}




\section{Introduction}

Fashion pressures and modern lifestyles, have encouraged women to wear high-heeled shoes $(\mathrm{HH})$. These fashion items have become the dominant choice of footwear for women, and are the preferred shoe of choice worn on many different occasions. High heeled shoes, while pleasant to look at, could be causing the wearer harm to the foot and lower limb. In spite of numerous opinions that oppose the use of $\mathrm{HH}$, these fashion items still remain highly popular among the female population. Several surveys have identified that between $37 \%$ to $69 \%$ of women wear HH on a daily basis [1]. It has also been suggested that wearing $\mathrm{HH}$ can change the force distribution of the foot and have negative effect on foot structure and morphology. The greater pressure observed might affect the foot segments involved in controlling the whole body during the standing phase and throughout motion. This noticeable at the first metatarsal where shear force, ground reaction force and loading rate significantly increase when wearing $\mathrm{HH}[2,3]$. This abnormally high force increase during landing changes the function of the foot. Research has shown that wearing $\mathrm{HH}$ on a regular basis leads to foot deformities and injuries that include metatarsal pain, Achilles tendon tension, plantar fasciitis and Haglund malformation [4]. In addition, a survey of high heeled shoe wearers has shown that the foot pain usually occurs in the toes, the hind foot and arch [5]. In spite of these research findings, there is still not enough evidence to explain the specific mechanisms associated with foot injury, deformity and disease that occurs from wearing $\mathrm{HH}$. Also, research focusing on the understanding of the kinematic effects on the foot when wearing $\mathrm{HH}$ is currently limited, therefore additional work is needed to explore this further [6].

The kinematic multitudinous literature has outlined the effects of high heels on the lower limbs. This research, has focused on the hip, knee and ankle, and has investigated the associated changes in the angles of these joints in three different planes during motion. The inner movement mechanisms have been identified when wearing high heels in these specific joints. However, kinematic details of the effects of wearing high heels on the dynamics and the structure of the foot are limited. Yu et al. [7] has suggested that the force distribution changes when wearing high heels and contributes to hallux valgus in forefoot. Theoretically, foot posture consists of alignment of the foot skeleton, and any abnormal landings or misalignments will change posture and affect the function of the foot directly. 
Data relating to the impact of high heels on foot kinematics is needed using a more reliable and objective classification method. Mechanical/biomechanical performance evaluation when wearing high heels, can be achieved using three-dimensional kinematics. This technique may provide a more in-depth analysis of foot mechanics while outlining greater structural detail.

The Oxford foot model (OFM) is a multi-segment model and provides comprehensive foot movement details that can be analysed to reveal the mechanical structure of the hallux, forefoot and hind foot during gait $[8,9]$. The repeatability and reliability of the Oxford foot model has been outlined in many biomechanical studies and has been widely used, particularly in experiments investigating foot movement $[10,11]$. In order to better understand the function of the flatfoot and to evaluate any degree of deformation, researchers have used the Oxford foot model to explore the characteristics of the flat-arched foot during gait analysis. Using the OFM, the different flatfoot features were revealed. The findings outlined that movement of the forefoot and hind foot in the dimensional space were significantly different from the normal foot. This study provided additional scientific support for the study of flatfoot pathology $[12,13]$. In addition, further details of foot movement were analyzed using the Oxford foot model that provided useful information to alleviate foot deformation in children $[14,10]$. In this context, the OFM can also be used to explore the complicated motion characteristics of the foot when subjects are wearing high heels. There have been no systematic studies published investigating high heel effects on foot mechanics using the Oxford Food Model. It has been reported that wearing high-heeled shoes can directly affect foot movement characteristics, such as the hallux, metatarsophalangeal joint and the hind foot. In relation to this, a more accurate and objective measurement of the foot position is needed when analysing and evaluating the kinematics and potential influence of wearing high heels on the feet.

The Oxford foot model has not been used to evaluate mechanical characteristics of the foot in individuals wearing high heeled shoes. Therefore, the purpose of this study was to investigate changes in the hallux, forefoot and hind foot motions when wearing high heels using the Oxford Foot Model. A further aim was to compare any kinematic differences between barefoot subjects and wearing high heels during gait analysis. 


\section{Method}

\subsection{Subjects}

Fifteen healthy women $(23+2.5$ years, $1.65+0.3 \mathrm{~m}, 51+3.6 \mathrm{~kg})$ were recruited from Ningbo University. Prior to testing, individual subject foot posture were scanned to ensure normality of the foot. In addition, all participants reported as having no previous lower extremity damage, foot diseases or deformities. The subjects in this study were all inexperienced high heeled wearers. Prior to commencement of the experiment, all subjects were clearly informed of experimental purpose, procedure and process, and all provided written informed consent. The experiment was granted Ethical approval by the Ethics Committee of the University (RAGH20171215).

\section{2 Equipment and procedure}

A Vicon motion system with 8 cameras (Oxford Metrics Ltd., Oxford, UK) was used to capture kinematic data of the hallux, forefoot and hind foot using a frequency at $100 \mathrm{HZ}$. The study was conducted under two different experimental conditions, walking barefoot (BF) versus wearing standard high heels of $5 \mathrm{~cm}(\mathrm{HH})$. The foot size of experimental shoes ranged from 36-38. Data was

collected from their dominant foot. To evaluate the three-dimensional movement of the left foot, the reflective markers were placed according to guidelines outlined in Plug in Gait as defined by Stebbins et al [11]. The OFM markers attached on the right leg were based on a previous definition (Fig 1). In order to define the hallux segment, a marker was placed (HLX) on the hallux, on the proximal end of the distal phalanx, or the distal end of the medial phalanx. The forefoot segment was identified by markers placed on the base of the $1^{\text {st }}$ metatarsal $(\mathrm{P} 1 \mathrm{M})$, and markers on the base of the $5^{\text {th }}$ metatarsal $(\mathrm{P} 5 \mathrm{M})$. The D1M marker was placed on the head of the $1^{\text {st }}$ metatarsal, and the D5M marker on the head of the $5^{\text {th }}$ metatarsal. This was completed ensuring that they were all the same distance from the plantar surface of the foot. The hind foot segment was defined by placing the MMA marker on the medial malleoli, with the LCA and STL markers on the lateral and medial aspects of the calcaneus respectively. The HEE marker was placed on the line that bisected the calcaneus, and the CPG marker was placed on the same line, above the HEE marker. The PAC marker was located on the same line, above the base of the CPG marker. To define the tibia segment, the marker (TUB) was placed on the tibial tuberosity, attached to the HFB marker on the head of the fibula, palpating the landmark from inferior to superior. The SHN marker was placed on 
the anterior crest of the tibia. To determine the position of the pelvis, the markers were attached to the anterior superior iliac spine (RASI), posterior superior iliac spine (RPSI) and a sacral marker attached midway between the posterior superior iliac spines (SACR). The markers for the pelvis and right lower limb are shown in Table 1 and Fig 1.

Following warm up, the subjects with markers were asked to stand in a suitable data capture position in front of the camera for static data collection. Once dynamic testing was performed, the three markers (RMMA, RPCA, RD1M) were removed [8]. Participants were asked to walk through the data capture area at a normal speed. The subjects were tested five times to ensure gait stability and to reduce experimental data collection error. All subjects completed the study twice, barefoot and wearing high heeled shoes on two separate days. Subjects were randomly assigned to both experimental conditions.

Figure 1 near here.

Table 1 near here.

\subsection{Data analysis}

The timing and magnitude of angular movement of the hallux with forefoot, forefoot with respect to hind foot, hind foot with respect to tibia during a complete gait cycle were compared between barefoot and wearing high heels in a sagittal plane, a frontal plane and a transverse plane respectively. Statistical analysis was performed using statistical software SPSS 19.0 (SPSS Inc,

Chicago, IL, USA). In order to assess any differences in kinematic parameters between the barefoot and high heeled conditions, independent sample t-tests were used. For all analysis the significance level was set at 0.05 .

\section{Results}

Peak values for angular motion for hallux relative to forefoot in all three planes, for barefoot and when wearing high heels are shown in Fig2 (a). Walking with high heeled shoes demonstrated 
significantly greater hallux dorsiflexion during the last stance phase $\left(22.55^{\circ} \pm 1.62^{\circ} \mathrm{VS} 26.6^{\circ} \pm 2.33^{\circ}\right.$ for the barefoot; $\mathrm{P}=0.001$ ). In addition, high heels showed less hallux plantarflexion during the initial stance phase $\left(-4.86^{\circ} \pm 2.32^{\circ} \mathrm{VS}-8.68^{\circ} \pm 1.13 ; \mathrm{P}<0.001\right)$ (see Table 2 ).

In all three planes of motion the kinematics of the forefoot relative to the hind foot are presented in Fig2 (b). Walking with high heeled shoes significantly increase forefoot abduction compared to barefoot walking during the late stance $\left(16.15^{\circ} \pm 1.37^{\circ}\right.$ VS $\left.13.18^{\circ} \pm 0.79^{\circ} ; \mathrm{P}<0.001\right)$. Compared to the barefoot condition, the forefoot adduction angle when wearing high heels was not statistically different. Furthermore, there were no significant differences found between the sagittal and frontal planes (see Table 2).

For all three planes of motion, the kinematic data of the hind foot relative to the tibia are outlined in Fig2 (c). In contrast to barefoot, the high heeled values show larger dorsiflexion in the horizontal plane during the terminal stance phase $\left(16.59^{\circ} \pm 1.69^{\circ} \mathrm{VS} 12.08^{\circ} \pm 0.9^{\circ} ; \mathrm{P}<0.001\right)$, but no differences were found in plantarflexion. Additionally, in the frontal plane, high heeled wearers showed greater angle of internal rotation than barefooted subjects during the initial stance phase $\left(16.72^{\circ} \pm 0.48^{\circ} \mathrm{VS} 7.97^{\circ} \pm 0.55^{\circ} ; \mathrm{P}<0.001\right)$. Also, the HH presented significantly less extension rotation compared to $\mathrm{BF}$ during the mid-stance phase $\left(-5.49^{\circ} \pm 0.69^{\circ} \mathrm{VS}-10.73^{\circ} \pm 0.42^{\circ}\right)$, but there were no significant differences recorded in the transverse plane (see Table 2).

Table 2 near here.

Figure 2 near here.

\section{Discussion}

This was first study to use the Oxford foot model to investigate changes in foot kinematics with and without high heels during walking. The hallux, forefoot and hind foot kinematics during gait were analysed. The results from this study showed that there were significant differences in sagittal plane motion of the hallux between BF and $\mathrm{HH}$ during gait analysis. 
There is a general opinion that wearing high heels leads to unfavourable load conditions that have detrimental effects on the structure of the foot [15]. In order to maintain gait stability, the larger sagittal plane dorsiflexion may be compensated by increased duration and amplitude of the ankle extensor moment, which increases the load on the ankle. This observation is consistent with the findings of Cronin and Barrett [16]. These factors greatly increase the risk of ankle injury. Healey et al. [17] observed that when wearing HH the hallux dorsiflexion increased significantly, and would lead to a shortening of the metatarsal fascia. This was related to the winch mechanism which causes the medial longitudinal arch to rise due to the traction of the metatarsal fascia. Previous research has stated that when wearing high heels, the peak pressure and pressure time integral increased in the hallux $[18,19]$. The results of this experiment demonstrated that the hallux presented higher dorsiflexion when wearing high heels and indicated that there was longer stress times recorded. This may explain in part why there is a greater pressure time integral in the hallux. The greater force observed and longer times spent in this position, would increase the risk of pain and malformation.

In the transverse plane of movement, the forefoot abduction maximum value increased during the last stance (toe off) was found to be higher for the high heeled condition compared to barefoot. Wearing high heels caused the foot to slide towards the head of the shoe. This resulted in squeezing between the shoes and the forefoot, and was problematic as the head of the high heel shoes were narrower than the foot. This had a major effect on the first metatarsal head, and this was extruded inwards significantly. Therefore, the peak value for abduction increased in the forefoot when wearing $\mathrm{HH}$. The abduction of the forefoot was a part of the foot supination-external rotation during the terminal stance. This increase might impact on the foot and cause hallux valgus. These two measures have been observed to be highly correlated. Hallux valgus is defined as a common foot abnormality which is defined as the hallux angle being deviated more than $15^{\circ}$ toward the lesser toes relative to the first metatarsal head [20]. McBride at al. [15] stated that when wearing high heeled shoes greater GRF are recorded at the first metatarsal than observed in barefoot conditions. In addition, the loading rate, shear stress and force concentration have also been observed to increase remarkably. This change in distribution of force in the forefoot has been thought to be correlated with foot deformities such as hallux valgus [21]. This study provided kinematic evidence that wearing high heels can lead to hallux valgus. The study further revealed the potential internal 
mechanism which caused foot deformity.

In the present study, compared to $\mathrm{BF}$, when walking with $\mathrm{HH}$ we observed that in the hind foot dorsiflexion was significantly increased and plantarflexion decreased. This may be an important potential factor in restricting the range of motion of the ankle in the kinetic chain. Cronin et al. [22] suggested that Achilles tendon force increased when wearing high heels shoes and caused Achilles tendon stiffness. The research identified that when wearing high heels, there was a significant increase in hind foot dorsiflexion that may contribute to maintenance of body balance and gait stability which was compensated by an increase in Achilles tendon stiffness. However, Achilles tendon stiffness could be attributable to a reduction the range of movement at the ankle joint [23]. Therefore, wearing $\mathrm{HH}$ for a long time periods can lead to Achilles pain and increases the risk of ankle sprain. Furthermore, the results of this study suggest that (Table 2), compared to the BF condition, wearing $\mathrm{HH}$ showed larger internal rotation. In anatomical terms the talus provides close links between the hind foot and the tibia. It is possible therefore to speculate that the internal rotation increase in the hind foot might also lead to an increase in the internal rotation of the tibia. Also, there is a coupling movement relationship between the subtalar joint and the lower limb [24]. If the hind foot motion was changed, this may affect the movement of the tibia and therefore would have consequences for the function of the proximal joints, such as knee [25]. Simonsen et al. [26] suggested that the arches were raised when wearing high heels, and that ankle plantarflexion was significantly increased. This would restrict ankle eversion, and any compensatory motion would increase the inversion of hind foot. However, the results from this study have indicated that there no significant differences in measures on inversion between the $\mathrm{HH}$ and the $\mathrm{BF}$ conditions (Table 2).

Conversely, the kinematic model of the foot and classification method of posture is very different between studies which makes the results difficult to compare [27]. In previous studies, the OFM has been used to evaluate different populations which can prove valid repeatability measures for the hallux, the forefoot and hind foot segment [8]. The midfoot has a very important role in motion transfer from the forefoot to the hind foot, but in this study movement in the midfoot was not detected. A further finding was that the height variation of the arch was not evaluated, and high heels can damage the natural arch form which increase the risk of arch injury. Baker et al. [28] suggested that a more detailed analysis is needed on the kinematics of the foot. Therefore, in order 
to make further conclusions about the effects of high heels on foot morphology and biomechanics, further specific research is required.

\section{Conclusion}

The OFM has revealed the kinematic and biomechanical details of the foot when wearing high heels during the walking. Subjects wearing high heels showed greater peak dorsiflexion and smaller plantarflexion of the hallux, and greater abduction of the forefoot. These findings support the opinion that wearing high heels can lead to hallux valgus. This is the first time that the intrinsic detailed mechanism of wearing high heels has been recorded, and provides details of how the foot is affected. This dataset on foot kinematics during walking gait provide an important basis for the explanation of foot deformity, foot disease and knee osteoarthritis in relation to wearing high heeled shoes. 


\section{References}

[1] Pazit Levinger, George S. Murley, Christian J. Barton. A comparison of foot kinematics in people with normal- and flat-arched feet using the Oxford Foot Model. Gait \& posture. 2010; 32:519.

[2]Cong Y, Cheung JT, Leung AK, Zhang M. Effect of heel height on in-shoe localized triaxial stresses. Journal of Biomechanics. 2011; 44:2267-2272.

[3]YungHui L, WeiHsien H. Effects of shoe inserts and heel height on foot pressure, impact force, and perceived comfort during walking. Applied ergonomics. 2005; 36:355.

[4]Ebbeling CJ, Hamill J, Crussemeyer JA. Lower extremity mechanics and energy cost of walking in high-heeled shoes. Journal of Orthopaedic \& Sports Physical Therapy. 1994; 19:190.

[5]American Podiatric Medical Association. High Heels Survey: 2003.

[6]Cronin NJ. The effects of high heeled shoes on female gait: a review. Journal of the International Society of Electrophysiological Kinesiology. 2014; 24:258.

[7]Yu J, Cheung TM, Fan Y, Zhang Y, Leung KL, Zhang M. Development of a finite element model of female foot for high-heeled shoe design. Clinical Biomechanics. 2008; 23:31.

[8]Carson MC, Harrington ME, Thompson N, Connor JJ, Theologis TN. Kinematic analysis of a multi-segment foot model for research and clinical applications: a repeatability analysis. Journal of Biomechanics. 2001; 34:1299-1307.

[9]Grood ES, Suntay WJ. A Joint Coordinate System for the Clinical Description of ThreeDimensional Motions: Application to the Knee. Journal of Biomechanical Engineering. 1983; 105:136-44.

[10]Curtis DJ, Bencke J, Stebbins JA, Stansfield B. Intra-rater repeatability of the Oxford foot model in healthy children in different stages of the foot roll over process during gait. Gait \& Posture. 2009; 30:118.

[11]Stebbins J, Harrington M, Thompson N. Repeatability of a model for measuring multi-segment foot kinematics in children. Gait \& Posture. 2006; 23:401-10. 
[12]Levinger P, Murley GS, Barton CJ, Cotchett MP, Mcsweeney SR, Menz HB. A comparison of foot kinematics in people with normal- and flat-arched feet using the Oxford Foot Model. Gait \& posture. $2010 ; 32: 519$.

[13]Matthias Hösl, Harald Böhm, Christel Multerer. Does excessive flatfoot deformity affect function? A comparison between symptomatic and asymptomatic flatfeet using the Oxford Foot Model. Gait \& Posture. 2014; 39:23-28.

[14]Mindler GT, Kranzl A, Lipkowski CA., Ganger R, Radler C. Results of gait analysis including the oxford foot model in children with clubfoot treated with the ponseti method. Journal of Bone \& Joint Surgery American Volume. 2014; 96:1593.

[15]Cavanagh PR, Morag E, Boulton A J, Young MJ, Deffner KT, Pammer SE. The relationship of static foot structure to dynamic foot function. Journal of Biomechanics. 1997;30: 243-250.

[16]Mcbride ID, Wyss UP, Cooke TD, Murphy L, Phillips J, Olney SJ. Long-term use of highheeled shoes alters the neuromechanics of human walking. Journal of applied physiology. 2012; $112: 1054$.

[17]Healey K, Chen K. Plantar Fasciitis: Current Diagnostic Modalities and Treatments. Clinics in Podiatric Medicine \& Surgery. 2010; 27:369-380.

[18]Snow RE, Williams KR, Jr HG. The effects of wearing high heeled shoes on pedal pressure in women. Foot \& Ankle. 1992; 13:85.

[19]Speksnijder CM, Munckhof RJ, Moonen SAF, Walenkamp GHI. The higher the heel the higher the forefoot-pressure in ten healthy women. Foot. 2005; 15:17-21.

[20]Nguyen US, Hillstrom HJ, Li W, Dufour AB, Kiel DP, Procter-Gray E, et al. Factors associated with hallux valgus in a population-based study of older women and men: the MOBILIZE Boston Study. Osteoarthritis \& Cartilage. 2010; 18:41-46.

[21] Hong WH, Lee YH, Chen HC, Pei YC, Wu CY. Influence of heel height and shoe insert on comfort perception and biomechanical performance of young female adults during walking. Foot \& Ankle International. 2005; 26:1042-1048. 
[22]Csapo R, Maganaris CN, Seynnes OR, Narici MV. On muscle, tendon and high heels. Journal of Experimental Biology. 2010; 213:2582.

[23] Cronin NJ, Peltonen J, Ishikawa M, Komi PV, Avela J, Sinkjaer T. Effects of contraction intensity on muscle fascicle and stretch reflex behavior in the human triceps surae. Journal of Applied Physiology. 2008; 105:226.

[24]Lundberg A, Goldie I, Kalin B, Selvik G. Kinematics of the ankle/foot complex: plantarflexion and dorsiflexion. Foot \& Ankle. 1989; 9:194.

[25]Williams DS, Mcclay IS, Hamill J, Buchanan TS. Lower Extremity Kinematic and Kinetic Differences in Runners With High and Low Arches. Journal of Applied Biomechanics. 2001.

[26] Simonsen OH, Revald P, Kjaer IL, Christensen M, Molgaard C, Lass P. Tibialis posterior tendon dysfunction. An often neglected cause of painful adult flatfoot. Ugeskrift for Laeger. 2006; 168:3314-6.

[27]McGinley JL, Baker R, Wolfe R, Morris ME. The reliability of three-dimensional kinematic gait measurements: a systematic review. Gait \& posture. 2009; 29:360.

[28] Balker R, Robb J. Foot models for clinical gait analysis. Gait Posture. 2006; 23:399-400. 
Table 1: Marker description of the Oxford foot model

\begin{tabular}{|c|c|}
\hline Markers & Description- Position \\
\hline RASI & Anterior Superior illiac Spine \\
\hline RPSI & Posterior Superior iliac Spine \\
\hline SACR & $\begin{array}{l}\text { Sacral marker- midway between the posterior } \\
\text { superior iliac spines }\end{array}$ \\
\hline RKNE & Standard lateral knee \\
\hline RTIB & Tibia marker \\
\hline RHFB & Later head of fibula \\
\hline RTUB & Tibia tuberosity \\
\hline RSHN & Anterior aspect of the skin \\
\hline RANK & Ankle \\
\hline RMMA & Medial Malleoli \\
\hline RCPG & Peg marker-Posterior end ot the calcaneus \\
\hline RHEE & Heel \\
\hline RACA & Posterior calcaneus proximal \\
\hline RLCA & Lateral calcaneus \\
\hline RSTL & Sustaniculum Tail \\
\hline RP1M & $1^{\text {st }}$ metatarsal, proximal dorsal \\
\hline RD1M & $1^{\text {st }}$ metatarsal, distal medial \\
\hline RP5M & 5st metatarsal, proximal lateral \\
\hline RD5M & 5st metatarsal, distal lateral \\
\hline RTOE & Toe \\
\hline RHLX & Hallux \\
\hline
\end{tabular}


Table 2. The peak value of angular motion for hallux relative to forefoot, forefoot relative to hindfoot and hindfoot relative to tibia in the barefoot and high heels shoes.

\begin{tabular}{|c|c|c|c|}
\hline Variable & $\begin{array}{c}\text { BF (deg) } \\
(\text { Mean } \pm \text { SD) }\end{array}$ & $\begin{array}{c}\mathrm{HH}(\mathrm{deg}) \\
(\text { Mean } \pm \mathrm{SD})\end{array}$ & P Value \\
\hline \multicolumn{4}{|l|}{ Hallux relative to forefoot } \\
\hline Dorsiflexion & $22.55 \pm 1.62$ & $26.6 \pm 2.33$ & 0.001 \\
\hline Plantar- flexion & $-8.68 \pm 1.13$ & $-4.86 \pm 2.32$ & $<0.001$ \\
\hline \multicolumn{4}{|l|}{ Forefoot relative to hindfoot } \\
\hline Dorsiflexion & $12.49 \pm 0.45$ & $11.46 \pm 2.49$ & 0.7 \\
\hline Plantar- flexion & $-4.04 \pm 1.04$ & $-1.7 \pm 1.79$ & 0.05 \\
\hline Abduction & $13.18 \pm 0.79$ & $16.15 \pm 1.37$ & $<0.001$ \\
\hline Adduction & $8.42 \pm 2.81$ & $7.19 \pm 0.41$ & 0.15 \\
\hline Inversion & $4.16 \pm 1.67$ & $4.45 \pm 1.06$ & 0.11 \\
\hline Eversion & $-4.47 \pm 0.64$ & $-5.12 \pm 0.7$ & 0.86 \\
\hline \multicolumn{4}{|l|}{ Hindfoot relative to tibia } \\
\hline Dorsiflexion & $12.08 \pm 0.9$ & $16.59 \pm 1.69$ & $<0.001$ \\
\hline Plantar- flexion & $-16.26 \pm 1.93$ & $-18.1 \pm 2.29$ & 0.16 \\
\hline Inversion & $6.88 \pm 0.9$ & $8.16 \pm 1.16$ & 0.07 \\
\hline Eversion & $-14.4 \pm 1.69$ & $-15.92 \pm 0.41$ & 0.19 \\
\hline Internal rotation & $7.97 \pm 0.55$ & $16.72 \pm 0.48$ & $<0.001$ \\
\hline External rotation & $-10.73 \pm 0.42$ & $-5.49 \pm 0.69$ & 0.001 \\
\hline
\end{tabular}

Note: the significant at $\mathrm{P}<0.05$. 
Fig 1. Oxford Foot Model Markers position under barefoot and high heel conditions. 


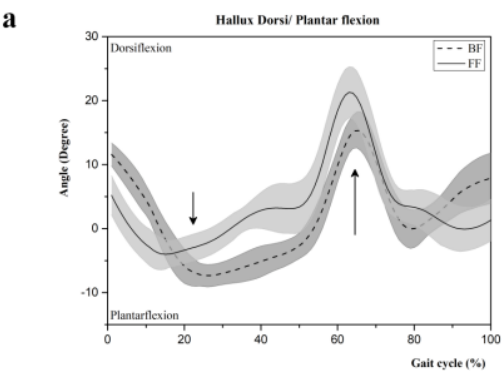

b
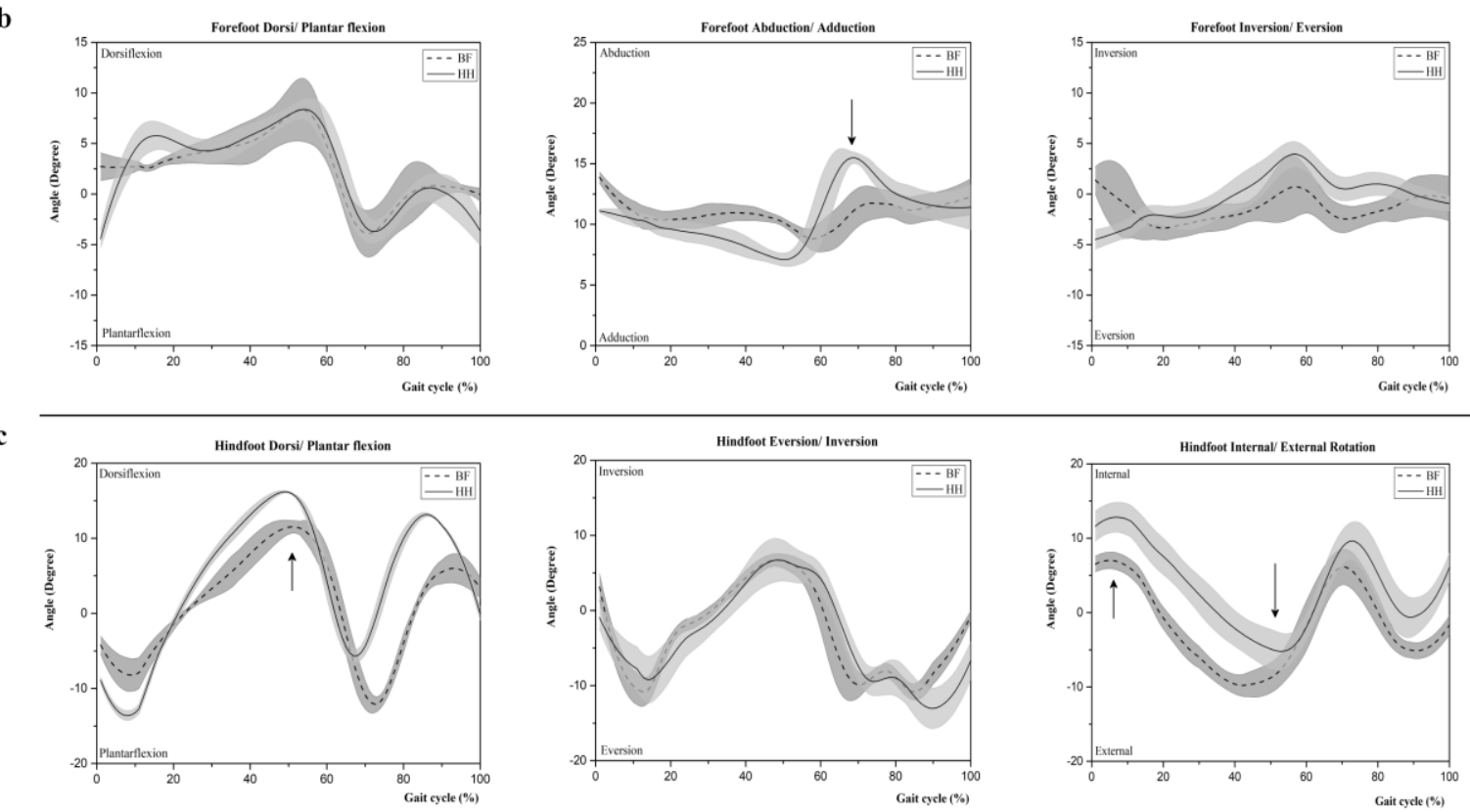

Fig 2. The foot kinematics $\left(^{\circ}\right)$ in three different plane during the gait cycle for high heels (HH) VS barefoot (BF). Arrows indicated the significant differences between $\mathrm{HH}$ and BF. The shaded band shows mean $\pm \mathrm{SD}$ in all controls.

a. Hallux relative to forefoot movement (degrees) in sagitta plane in subjects with $\mathrm{HH}$ and BF.

b. Forefoot relative to hindfoot motion (degrees) in sagitta, frontal and transverse planes in subjects with $\mathrm{HH}$ and $\mathrm{BF}$.

c. Hindfoot relative to tibia motion (degrees) in sagitta, frontal and transverse planes in subjects with $\mathrm{HH}$ and $\mathrm{BF}$. 